\title{
Optimal Randomization of Signal Constellations on the Downlink of a Multiuser DS-CDMA System
}

\author{
Mehmet Emin Tutay, Student Member, IEEE, Sinan Gezici, Senior Member, IEEE, \\ and Orhan Arikan, Member, IEEE
}

\begin{abstract}
In this study, the jointly optimal power control with signal constellation randomization is proposed for the downlink of a multiuser communications system. Unlike a conventional system in which a fixed signal constellation is employed for all the bits of a user (for given channel conditions and noise power), power control with signal constellation randomization involves randomization/time-sharing among different signal constellations for each user. A formulation is obtained for the problem of optimal power control with signal constellation randomization, and it is shown that the optimal solution can be represented by a randomization among $(K+1)$ or fewer distinct signal constellations for each user, where $K$ denotes the number of users. In addition to the original nonconvex formulation, an approximate solution based on convex relaxation is derived. Then, detailed performance analysis is presented when the receivers employ symmetric signaling and sign detectors. Specifically, the maximum asymptotical improvement ratio is shown to be equal to the number of users, and the conditions under which the maximum and minimum asymptotical improvement ratios are achieved are derived. Numerical examples are presented to investigate the theoretical results, and to illustrate performance improvements achieved via the proposed approach.
\end{abstract}

Index Terms-Multiuser, downlink, probability of error, timesharing, randomization, minimax.

\section{INTRODUCTION}

$\mathbf{I}$ $\mathrm{N}$ this study, we consider a generic problem on the signal constellation design for the downlink of a binary multiuser communications system in which users can randomize or timeshare among multiple signal constellations. Unlike conventional systems in which a fixed signal constellation is employed for all the bits of a user (for given channel conditions and noise power) [1], we formulate a generic problem that can involve randomization/time-sharing among different signal constellations for each user. Due to such randomization/timesharing, the signal amplitude corresponding to each bit of a user can be modeled as a generic random variable in this approach. Therefore, the problem can be formulated as obtaining the optimal probability distribution for the signal

Manuscript received September 17, 2012; revised May 27, 2013; accepted June 8,2013 . The associate editor coordinating the review of this paper and approving it for publication was A. Chockalingam.

The authors are with the Department of Electrical and Electronics Engineering, Bilkent University, Bilkent, Ankara, 06800, Turkey (e-mail: \{tutay, gezici, oarikan\}@ee.bilkent.edu.tr).

This research was supported in part by the National Young Researchers Career Development Programme (project no. 110E245) of the Scientific and Technological Research Council of Turkey (TUBITAK).

Part of this work was presented at the IEEE International Workshop on Signal Processing Advances in Wireless Communications (SPAWC), June 2012.

Digital Object Identifier 10.1109/TW.2013.081413.121418 amplitude corresponding to each bit of each user in a multiuser system.

The proposed approach, which is called power control with signal constellation randomization, differs from randomized power control algorithms in the literature from various perspectives [2]-[7]. ${ }^{1}$ First, as the power control with signal constellation randomization approach can result in strategies in which different power allocation strategies are employed for different bits of a given user, it is a more generic approach than randomized power control in general. Second, the proposed approach is employed for each state of the channel whereas power control algorithms are used with respect to varying channel conditions. In other words, the power control strategies in the literature adapt the power as the channel state changes, whereas the proposed approach performs constellation randomization for a given (fixed) channel state. Third, even for the symmetric signaling case (in which signal amplitudes for bit 0 and bit 1 are negatives of each other, and the same power allocation strategy is employed for bit 0 and bit 1 for each user), the proposed approach differs from those in the literature [2]-[7] by modeling the signal amplitudes (powers) of the users as generic random variables and obtaining the optimal probability distributions of those random variables that minimize a probability of error metric. For example, in [2], transmit powers are selected from a discrete set of power levels, namely, zero and peak power, and optimal power randomization strategies are obtained under that specification for a two-hop interference channel. ${ }^{2}$ [3] considers the same strategy for power control in ad-hoc sensor networks, and works on the optimization of transmission (on-state) probability to meet certain quality of service requirements. In another study [4], a random power control algorithm is proposed, in which the transmitter selects its power level randomly from a uniform distribution. It is shown that this approach can improve network connectivity over the fixed power control approach in the case of static channels. However, the performance of this uniform power selection approach deteriorates in fading channels, as investigated in [5]. In [6], random power allocation according to a certain probability distribution is proposed. Namely, the transmit power is modeled by a truncated inverted exponential distribution, and the parameter of this distribution is updated at certain intervals based on feedback. The connectivity analysis of this

\footnotetext{
${ }^{1}$ Please refer to [8]-[10] for surveys on power control in wireless networks.

${ }^{2}$ Please refer to [11] and [12] for other game theoretic approaches for power control.
} 
approach is presented in [7] for wireless sensor networks, and improvements in energy efficiency are observed. ${ }^{3}$

The power control with signal constellation randomization problem considered in this study is also related to some of the recent works in the literature that investigate the effects of randomizing transmitted signals, additive "noise", and jammer power [15]-[24]. In [15], the stochastic signaling approach is considered by modeling transmitted signal amplitudes in a binary communications system as random variables instead of fixed quantities for each information symbol. It is shown that the probability of error is minimized when each signal amplitude is represented by a randomization (time-sharing) of at most three different signal levels under second and fourth moment constraints. The results are extended in [16] to cases in which signals and detectors are jointly designed. In addition, [18] investigates the problem of joint detector randomization and stochastic signaling for minimum probability of error receivers. The effects of randomization are observed also in improving performance of suboptimal detectors and estimators by injecting "noise" to their observations [19]-[21], [25]. For example, additive noise that is a randomization between two different signal levels can increase detection probabilities of some suboptimal detectors under false-alarm constraints [19], [20]. The studies in [22] and [23] investigate the convexity properties of the average probability of error in the presence of additive white Gaussian noise (AWGN) when maximum likelihood (ML) detectors are employed at the receivers. Based on the convexity results, the cases in which power randomization can or cannot be useful for improving error performance are specified, and optimal jammer power randomization strategies are proposed. In [24], the jamming problem is studied based on a dynamic game model, in which transmit and jammer powers can be chosen randomly.

The idea of using time-varying or random signal constellations is considered in some studies such as [26]-[31]. In [26], the author proposes (pseudo)randomly rotating the signal constellation for each transmitted vector in order to improve the coded frame-error-rate of spatial multiplexing in block fading. The advantages of this approach in reducing the outage probability are investigated in [27]. Although a form of constellation randomization is performed in [26], [27], they are different from the current work since a (pseudo)random rotation of the signal constellation is proposed for a single user system in those studies, whereas we obtain optimal randomization of signal constellations for a multiuser system in this work. In addition, the studies in [28]-[31] consider random signal mapping, random rotations, or time-varying phase shifts to transmitted signals in order to achieve diversity.

Motivated by the recent results that illustrate the improvements obtained via randomization [15]-[26], the aim of this study is to formulate a generic power control problem with signal constellation randomization for the downlink of a multiuser communications system in which the signal amplitude for each bit of a user is modeled as a random variable. In other

\footnotetext{
${ }^{3}$ In [13] and [14], the term "stochastic power control" is used in a different meaning from "randomized power control" in [2]-[7]. Specifically, [13] and [14] do not employ any power or signal randomization but apply an approach that is based on measurements (which are inherently random) instead of known deterministic parameters.
}

words, by adopting the approach in [15], the aim is to jointly design the optimal randomization of signal constellations for all users in the downlink of a direct sequence code division multiple access (DS-CDMA) system in order to optimize error performance for given receiver structures. The main challenge in the joint design of signal constellation randomization is that signal amplitudes of each user affect not only its own error performance but also the performance of all other users via interference. The main contributions of this study can be summarized as follows:

- The joint design of optimal randomization of signal constellations is performed in the downlink of a multiuser system for given receiver structures.

- It is shown that the optimal power control with signal constellation randomization results in a randomization among up to $(K+1)$ different signal constellations for each user, where $K$ is the number of users.

- An approximate convex solution is obtained based on convex relaxation.

- Although the theoretical results are obtained for generic detector structures at the receivers (Section III), specific results are obtained for sign detectors (Section IV). Namely, it is shown that, in the absence of noise, the ratio between the maximum error probabilities of the optimal fixed signal constellations approach and the optimal power control with signal constellation randomization approach can be as high as the number of users.

In addition, numerical examples are provided to illustrate the improvements obtained via the proposed power control with signal constellation randomization approach (Section V).

\section{System Model}

Consider the downlink of a multiuser DS-CDMA binary communications system, in which the baseband model for the transmitted signal is given by

$$
p(t)=\sum_{k=1}^{K} S_{k}^{\left(i_{k}\right)} c_{k}(t)
$$

where $K$ is the number of users, $S_{k}^{\left(i_{k}\right)}$ denotes the amplitude of the $k$ th user's signal corresponding to bit $i_{k}$, with $i_{k} \in$ $\{0,1\}$, and $c_{k}(t)$ is the real pseudo-noise signal for user $k$. The pseudo-noise signals spread the spectra of users' signals and provide multiple-access capability [1]. Information intended for user $k$ is carried by $S_{k}^{\left(i_{k}\right)}$, which corresponds to bit 0 for $i_{k}=0$ and bit 1 for $i_{k}=1$. $S_{k}^{\left(i_{k}\right)}$,s are modeled as real numbers, and they scale the amplitudes of the pseudo-noise signals, $c_{k}(t)$ 's. It is assumed that bit 0 and bit 1 are equally likely (i.e., the prior probabilities of the bits are equal to 0.5 ) for all users, and the information bits for different users are independent.

The signal in (1) is transmitted to $K$ users, and the received signal at user $k$ is represented by

$$
r_{k}(t)=\sum_{l=1}^{K} S_{l}^{\left(i_{l}\right)} c_{l}(t)+n_{k}(t),
$$

for $k=1, \ldots, K$, where $n_{k}(t)$ denotes the noise at the receiver of user $k$, which is modeled as a zero-mean white 


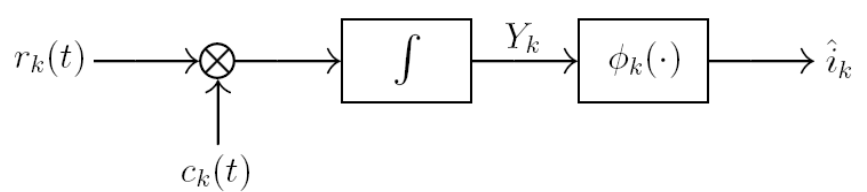

Fig. 1. Receiver structure for user $k$.

Gaussian process with spectral density $\sigma_{k}^{2}$. It is assumed that the noise processes at different receivers are independent. Although a simple additive noise model is employed in (2), multipath channels with slow frequency-flat fading can also be covered by the considered model if perfect channel estimation is assumed at the receivers [15]. (In that case, the average powers of the noise components in (2), equivalently, $\sigma_{k}^{2}$ terms, can be adjusted according to channel power gains in order to take the channel conditions into account.)

The receiver for user $k$ processes the signal in (2) as shown in Fig. 1. Specifically, the received signal $r_{k}(t)$ is correlated with the pseudo-noise signal for user $k, c_{k}(t)$, which effectively corresponds to a despreading operation, and then the correlator output is used by a generic detector in order to estimate the transmitted bit for user $k$. Based on (2), the correlator output for user $k$ can be expressed as

$$
Y_{k}=S_{k}^{\left(i_{k}\right)}+\sum_{\substack{l=1 \\ l \neq k}}^{K} \rho_{k, l} S_{l}^{\left(i_{l}\right)}+N_{k},
$$

for $k=1, \ldots, K$, where $\rho_{k, l} \triangleq \int c_{k}(t) c_{l}(t) d t$ denotes the crosscorrelation between the pseudo-noise signals for user $k$ and $l$ (it is assumed without loss of generality that $\rho_{k, k}=1$ for $k=1, \ldots, K)$, and $N_{k} \triangleq \int n_{k}(t) c_{k}(t) d t$ is the noise component. It can be shown that $N_{1}, \ldots, N_{K}$ form a sequence of independent zero-mean Gaussian random variables with variances, $\sigma_{1}^{2} \ldots, \sigma_{K}^{2}$, respectively. In (3), the first term corresponds to the desired signal component, the second term represents the multiple-access interference (MAI), and the last term is the noise component.

The correlator output in (3) is used by a generic detector (decision rule) $\phi_{k}$ to generate an estimate of the transmitted information bit, as shown in Fig. 1. Specifically, for a given correlator output $Y_{k}=y_{k}$, the bit estimate is denoted as

$$
\hat{i}_{k}=\phi_{k}\left(y_{k}\right)= \begin{cases}0, & y_{k} \in \Gamma_{k, 0} \\ 1, & y_{k} \in \Gamma_{k, 1}\end{cases}
$$

for $k=1, \ldots, K$, where $\Gamma_{k, 0}$ and $\Gamma_{k, 1}$ denote the decision regions for bit 0 and bit 1 , respectively, and they form a partition of the observation space [32]. In the next section, theoretical results are obtained for generic detectors at the receivers; that is, $\phi_{k}$ 's can be arbitrary decision rules.

\section{Power Control with Signal Constellation RANDOMIZATION FOR MULTIUSER Systems}

A. Optimal Power Control with Signal Constellation Randomization

In conventional systems, $S_{k}^{\left(i_{k}\right)}$ in (1) corresponds to a fixed value for each bit of a given user; in other words, a signal constellation is selected for each user, and it is employed for all the bits in the multiuser system (for given channel conditions and noise power). For example, consider a two-user system, in which bit 0 and bit 1 are represented by -1 and 1 , respectively, for user 1 , and by -0.5 and 0.5 , respectively, for user 2 . Then, the joint signal constellation for the two users is represented by $\left(S_{1}^{(0)}, S_{1}^{(1)}, S_{2}^{(0)}, S_{2}^{(1)}\right)=(-1,1,-0.5,0.5)$. In this case, there is no randomization or time-sharing among multiple signal constellations, and a fixed signal constellation is employed for all the bits of each user in the system for given channel conditions and noise power. A specific example is illustrated in Table I(A) when 12 bits are transmitted for each user.

Unlike conventional systems, we consider power control with signal constellation randomization in this study and model $S_{k}^{\left(i_{k}\right)}$ in (1) as generic random variables [15]. In this case, it is possible to employ different signal constellations for different bits in the system (for given channel conditions and noise power). In other words, randomization/time-sharing among multiple signal constellations is possible. For example, in a two-user system, one can time-share between joint signal constellations $\left(S_{1}^{(0)}, S_{1}^{(1)}, S_{2}^{(0)}, S_{2}^{(1)}\right)=(-0.7,0.7,-0.4,0.4)$ and $\left(S_{1}^{(0)}, S_{1}^{(1)}, S_{2}^{(0)}, S_{2}^{(1)}\right)=(-1.1,1.1,-0.8,0.8)$. Specifically, if half of the bits are sent according to the first set of signal constellations and the remaining half are sent according to the second one, the overall joint signal constellation, $\left(S_{1}^{(0)}, S_{1}^{(1)}, S_{2}^{(0)}, S_{2}^{(1)}\right)$, can be represented by a discrete random variable which is equal to $(-0.7,0.7,-0.4,0.4)$ or $(-1.1,1.1,-0.8,0.8)$ with equal probabilities. In Table I(B), this example of power control with signal constellation randomization is illustrated when 12 bit are transmitted for each user. As observed from the table, for user 1 (user 2), half of bits 0 are represented by $-0.7(-0.4)$ and the remaining half are represented by $-1.1(-0.8)$; similarly, half of bits 1 are represented by $0.7(0.4)$ and the remaining half are represented by $1.1(0.8)$ in order to implement the desired signal constellation randomization.

In order to provide a generic formulation of the proposed power control with signal constellation randomization approach in multiuser systems, let $\boldsymbol{S}$ denote the vector of random variables corresponding to the amplitudes of all users' signals for bit 0 and bit 1 ; that is,

$$
\boldsymbol{S}=\left(S_{1}^{(0)}, S_{1}^{(1)}, S_{2}^{(0)}, S_{2}^{(1)}, \cdots, S_{K}^{(0)}, S_{K}^{(1)}\right),
$$

where $S_{k}^{\left(i_{k}\right)}$ is as in (1). In other words, $\boldsymbol{S}$ is the joint signal constellation, which is a $2 K$ dimensional vector consisting of signal constellations for all users (as exemplified in the previous paragraphs), and it is modeled as a generic random vector in order to facilitate any type of signal constellation randomization. In addition, let $p_{\boldsymbol{S}}$ represent the probability density function (PDF) of $\boldsymbol{S}$. According to this definition, the conventional approach of no constellation randomization (or, fixed signal constellations) corresponds to a PDF in the form of $p_{\boldsymbol{S}}(\mathbf{s})=\delta\left(\mathbf{s}-\mathbf{s}_{0}\right)$, where $\delta(\cdot)$ represents the Dirac delta function. (For instance, $p_{\boldsymbol{S}}(\mathbf{s})=\delta(\mathbf{s}-(-1,1,-0.5,0.5))$ for the example in Table I(A).) On the other hand, any generic $P D F$ can be employed in the power control with signal constellation randomization approach considered in this study. 
TABLE I

(A) EXAMPLE OF A CONVENTIONAL SYSTEM IN WHICH NO SIGNAL CONSTELLATION RANDOMIZATION IS EMPLOYED. JOINT SIGNAL CONSTELLATION $\left(S_{1}^{(0)}, S_{1}^{(1)}, S_{2}^{(0)}, S_{2}^{(1)}\right)=(-1,1,-0.5,0.5)$ IS USED FOR ALL THE BITS. (B) EXAMPLE OF POWER CONTROL WITH SIGNAL CONSTELLATION RANDOMIZATION IN WHICH HALF OF THE BITS ARE TRANSMITTED ACCORDING TO JOINT SIGNAL CONSTELLATION $(-0.7,0.7,-0.4,0.4)$ AND THE REMAINING HALF ARE TRANSMITTED ACCORDING TO $(-1.1,1.1,-0.8,0.8)$.

(A)

\begin{tabular}{|c|c|c|c|c|c|c|c|c|c|c|c|c|}
\hline Bit of User 1 $\left(i_{1}\right)$ & 0 & 1 & 0 & 0 & 1 & 0 & 1 & 1 & 0 & 0 & 1 & 1 \\
Amplitude of User 1's Signal $\left(S_{1}^{\left(i_{1}\right)}\right)$ & -1 & 1 & -1 & -1 & 1 & -1 & 1 & 1 & -1 & -1 & 1 & 1 \\
\hline Bit of User 2 $\left(i_{2}\right)$ & 1 & 0 & 1 & 0 & 0 & 1 & 1 & 0 & 1 & 0 & 0 & 1 \\
Amplitude of User 2's Signal $\left(S_{2}^{\left(i_{2}\right)}\right)$ & 0.5 & -0.5 & 0.5 & -0.5 & -0.5 & 0.5 & 0.5 & -0.5 & 0.5 & -0.5 & -0.5 & 0.5 \\
\hline
\end{tabular}

(B)

\begin{tabular}{|c|c|c|c|c|c|c|c|c|c|c|c|c|}
\hline Bit of User 1 $\left(i_{1}\right)$ & 0 & 1 & 0 & 0 & 1 & 0 & 1 & 1 & 0 & 0 & 1 & 1 \\
Amplitude of User 1's Signal $\left(S_{1}^{\left(i_{1}\right)}\right)$ & -0.7 & 0.7 & -1.1 & -0.7 & 1.1 & -1.1 & 0.7 & 1.1 & -0.7 & -1.1 & 0.7 & 1.1 \\
\hline Bit of User 2 $\left(i_{2}\right)$ & 1 & 0 & 1 & 0 & 0 & 1 & 1 & 0 & 1 & 0 & 0 & 1 \\
Amplitude of User 2's Signal $\left(S_{2}^{\left(i_{2}\right)}\right)$ & 0.4 & -0.4 & 0.8 & -0.4 & -0.8 & 0.8 & 0.4 & -0.8 & 0.4 & -0.8 & -0.4 & 0.8 \\
\hline
\end{tabular}

(For instance, $p_{\boldsymbol{S}}(\mathbf{s})=0.5 \delta(\mathbf{s}-(-0.7,0.7,-0.4,0.4))+$ $0.5 \delta(\mathbf{s}-(-1.1,1.1,-0.8,0.8))$ for the example in Table I(B).)

Based on the definition in (5), the aim is to find the optimal PDF of $\boldsymbol{S}$, i.e., the optimal randomization of signal constellations, in a given multiuser system. Considering a generic approach in the sense that the PDF of $\boldsymbol{S}, p_{\boldsymbol{S}}$, can be in any form (corresponding to discrete, continuous, or mixed random variables), we formulate the following power control with signal constellation randomization problem:

$$
\begin{aligned}
& \min _{p_{S}} \max _{k \in\{1, \ldots, K\}} \mathrm{P}_{k} \\
& \text { subject to } \mathrm{E}\left\{\int|p(t)|^{2} d t\right\} \leq A
\end{aligned}
$$

where $\mathrm{P}_{k}$ denotes the average probability of error for user $k$, $p(t)$ is as in (1), and $A$ is a constraint on the average power of the transmitted signal. In other words, the aim is to find the optimal PDF for the joint signal constellation that minimizes the maximum of the average probabilities of error under a constraint on the average transmitted power. The minimax approach is adopted for fairness [33]-[38]; that is, for preventing scenarios in which the average probabilities of error are very low for some users whereas they are (unacceptably) high for others. Extensions to cases in which different users have different levels of importance are also possible as discussed in Section VI. It is noted that the formulation in (6)-(7) is similar to a max-min SINR problem [34]. However, the main differences are that the optimization in (6)-(7) is performed over the set of possible PDFs for the joint signal constellation, and that the considered probability of error metric leads to different solutions than the max-min SINR problem in general.

In order to express the optimization problem in (6)-(7) more explicitly, we first manipulate the average power expression in (7) based on (1) as follows:

$$
\mathrm{E}\left\{\int|p(t)|^{2} d t\right\}=\sum_{k=1}^{K} \sum_{l=1}^{K} \rho_{k, l} \mathrm{E}\left\{S_{k}^{\left(i_{k}\right)} S_{l}^{\left(i_{l}\right)}\right\}=\mathrm{E}\{H(\boldsymbol{S})\}
$$

where $H(\boldsymbol{S})$ is defined as

$$
H(\boldsymbol{S}) \triangleq \sum_{k=1}^{K} \sum_{l=1}^{K} \rho_{k, l} S_{k}^{\left(i_{k}\right)} S_{l}^{\left(i_{l}\right)}
$$

In some scenarios, symmetric signaling is used, that is, the amplitudes of users' signals corresponding to bit 0 and bit 1 are selected as $S_{k}^{(0)}=-S_{k}^{(1)}$ for $k=1, \ldots, K{ }^{4}$ In that case, $\mathrm{E}\left\{S_{k}^{\left(i_{k}\right)} S_{l}^{\left(i_{l}\right)}\right\}=\mathrm{E}\left\{\left|S_{k}^{(1)}\right|^{2}\right\}$ if $k=l$ and $\mathrm{E}\left\{S_{k}^{\left(i_{k}\right)} S_{l}^{\left(i_{l}\right)}\right\}=0$ if $k \neq l$ since information bits are equally likely. Then, $H(\boldsymbol{S})$ in (9) becomes $H(\boldsymbol{S})=\sum_{k=1}^{K}\left|S_{k}^{(1)}\right|^{2}$.

Next, the average probability of error for user $k, \mathrm{P}_{k}$, is obtained as follows (please see Appendix A for details):

$$
\mathrm{P}_{k}=\mathrm{E}\left\{G_{k}(\boldsymbol{S})\right\},
$$

where the expectation is over the random vector $S$ in (5), and $G_{k}(\boldsymbol{S})$ is defined as

$$
\begin{aligned}
G_{k}(\boldsymbol{S}) \triangleq \frac{1}{2^{K}} & \sum_{m \in\{0,1\}} \sum_{\mathbf{i}_{k} \in\{0,1\}^{K-1}} \mathrm{P}\left\{\left(N_{k}+S_{k}^{(m)}\right.\right. \\
& \left.\left.+\sum_{\substack{l=1 \\
l \neq k}}^{K} \rho_{k, l} S_{l}^{\left(i_{l}\right)}\right) \in \Gamma_{k, 1-m} \mid \boldsymbol{S}\right\} .
\end{aligned}
$$

The probabilities in (11) are calculated with respect to the PDF of $N_{k}$ for given values of $S_{k}^{\left(i_{k}\right)}$,s, and $\mathbf{i}_{k}$ is defined as $\mathbf{i}_{k} \triangleq$ $\left[i_{1} \cdots i_{k-1} i_{k+1} \cdots i_{K}\right]$; i.e., the vector of all the bit indices except for the $k$ th one. In (11), we consider fixed (given) decision rules at the receivers; that is, the decision regions, $\Gamma_{k, 1-m}$ 's, are independent of $p_{\boldsymbol{S}}$.

Based on (8) and (10), the optimization problem in (6)-(7) can be stated as

$$
\begin{aligned}
\min _{p_{S}} & \max _{k \in\{1, \ldots, K\}} \mathrm{E}\left\{G_{k}(\boldsymbol{S})\right\} \\
& \text { subject to } \mathrm{E}\{H(\boldsymbol{S})\} \leq A .
\end{aligned}
$$

The optimization problem in (12)-(13) can be quite complex in its current form since it requires optimization over all possible PDFs for a random vector of size $2 K$ (see (5)). ${ }^{5}$ However, various approaches can be taken in order to provide a simpler formulation of the optimization problem. To that end, the following proposition is presented first.

\footnotetext{
${ }^{4}$ For the example in Table I, symmetric signaling is employed.

${ }^{5}$ The dimension of vector $S$ can be reduced to $K$ if symmetric signaling is employed.
} 
Proposition 1: Suppose $G_{k}$ 's are continuous functions and the elements of $\boldsymbol{S}$ take values from finite closed intervals. Then, an optimal solution to (12)-(13) can be expressed as

$$
p_{\boldsymbol{S}}(\mathbf{s})=\sum_{j=1}^{K+1} \lambda_{j} \delta\left(\mathbf{s}-\mathbf{s}_{j}\right),
$$

where $\sum_{j=1}^{K+1} \lambda_{j}=1$ and $\lambda_{j} \geq 0$ for $j=1, \ldots, K+1$.

Proof: Please see Appendix B.

Proposition 1 states that an optimal joint signal constellation $S$ can be represented as a discrete random variable which corresponds to a randomization of $(K+1)$ or fewer distinct signal constellations for each user. In other words, for each information bit of each user, an optimal solution can be obtained by performing randomization among up to $(K+1)$ different signal amplitudes. This is unlike the conventional case in which a fixed amplitude value is transmitted for each information bit of a user.

Another implication of Proposition 1 can be provided as follows. Since a generic formulation is considered, the set of $G_{k}$ 's and $H$ corresponding to all possible joint signal constellations is not a convex set in general. Hence, the optimal solution of (12)-(13) can require randomization (timesharing), as expressed in (14), in order to achieve the points on the convex hull of this set. (Please see the proof of the proposition in Appendix B for a mathematical statement of this observation.)

In practice, randomization of signal constellations can be performed, for example, via time-sharing by employing each signal constellation for a certain number of information bits in proportion to the probability of that constellation. A simple example was provided in the second paragraph of this section and in Table I(B). More generally, if $N_{\mathrm{I}}$ information bits are to be transmitted to each user, $\lambda_{1} N_{\mathrm{I}}$ bits are generated according to $\mathbf{s}_{1}, \lambda_{2} N_{\mathrm{I}}$ bits are generated according to $\mathbf{s}_{2}, \ldots$, and $\lambda_{K+1} N_{\mathrm{I}}$ bits are generated according to $\mathbf{s}_{K+1}$ in order to realize the PDF of the joint signal constellation in (14). It should be emphasized that the receivers do not need to know this randomization structure since the signal constellation randomization is optimized by the transmitter for fixed (given) detectors at the receivers of different users (see (4)) based on the optimization problem in (6)-(7). (In particular, the average probability of error for user $k, \mathrm{P}_{k}$, in (6) is given by (10) and (11), which indicate that the decision regions $\Gamma_{k, 0}$ and $\Gamma_{k, 1}$ (equivalently, the detector) for each user are independent of the probability distribution of the joint signal constellation, $\boldsymbol{S}$; hence, the receiver implements its detector without knowing the randomization structure.)

Proposition 1 implies that it is not necessary to search over all PDFs in (12)-(13). Instead, only the PDFs in the form of (14) can be considered, and the problem in (12)-(13) can be reduced to

$$
\begin{aligned}
\min _{\left\{\lambda_{j}, \mathbf{s}_{j}\right\}_{j=1}^{K+1}} \max _{k \in\{1, \ldots, K\}} & \sum_{j=1}^{K+1} \lambda_{j} G_{k}\left(\mathbf{s}_{j}\right) \\
\text { subject to } & \sum_{j=1}^{K+1} \lambda_{j} H\left(\mathbf{s}_{j}\right) \leq A, \sum_{j=1}^{K+1} \lambda_{j}=1 \\
& \lambda_{j} \geq 0, j=1, \ldots, K+1
\end{aligned}
$$

Since this optimization problem is over a number of variables instead of functions, it provides a significant simplification over the problem in (12)-(13). However, it can still be a nonconvex optimization problem in general. The structure of the optimization problem in (15)-(16) can be utilized in order to obtain close-to-optimal solutions with low complexity. Namely, as discussed in the next subsection, a convex relaxation approach can be employed to provide an approximate solution of (15)-(16).

Remark: In order to realize the proposed approach of power control with signal constellation randomization in practice, the transmitter needs to know the noise powers at the receivers (or, the signal-to-noise ratios (SNRs) at the receivers, considering a flat-fading scenario, as discussed after (2)), which can be sent via feedback to the transmitter. Such a feedback is commonly available in multiuser systems for power control purposes [8]. In addition, if the randomization is implemented via time-sharing, the channel conditions should be (almost) constant for a number of bit durations; hence, slowly fading channels are well-suited for the power control with signal constellation randomization approach.

1) Power Control with Constellation Randomization versus Conventional Power Control: The main difference of the proposed power control with constellation randomization approach from conventional power control algorithms is that the former is employed for each state of the channel whereas the latter is used with respect to varying channel conditions. In other words, the power control strategies in the literature adapt the power as the channel state changes, whereas the proposed approach performs constellation (power) randomization for a given (fixed) channel state. Therefore, these two approaches are different in the sense that they are employed in different scenarios. In addition, it is possible to employ these two approaches jointly: conventional power control as the channel conditions change, and power control with constellation randomization for each channel state. In such a scenario, the conventional power control strategy will determine the power that is allocated for each channel state, which in effect sets the value of $A$ in (7), and the proposed approach will employ the optimal constellation randomization under the power limit based on the optimization problem in (6)-(7). Therefore, the proposed power control with constellation randomization approach is well-suited for slow fading channels, where the channel state is (almost) constant for a certain number of bit durations and then changes to a different value after a certain amount of time (i.e., block fading scenarios).

\section{B. Approximate Solution Based on Convex Relaxation}

Although the optimization problem in (15)-(16) can be solved via global optimization techniques in general, it becomes challenging for an optimization technique to achieve the global optimum as the number $K$ of users increases. ${ }^{6}$ Therefore, it is desirable to obtain a convex version of the problem, which always converges to its global optimum. In the following, an approximate formulation of the problem is provided based on convex relaxation [39].

${ }^{6}$ Specifically, there are a total of $(2 K+1)(K+1)$ unknown variables in (15)-(16) (which reduces to $(K+1)^{2}$ for symmetric signaling). 
First, consider a set of possible joint signal constellations for $\boldsymbol{S}$ in (5) and denote them as $\tilde{\mathbf{s}}_{1}, \ldots, \tilde{\mathbf{s}}_{N_{m}}$. Then, the PDF of the joint signal constellation is approximately modeled as

$$
p_{\boldsymbol{S}}(\mathbf{s}) \approx \sum_{j=1}^{N_{m}} \tilde{\lambda}_{j} \delta\left(\mathbf{s}-\tilde{\mathbf{s}}_{j}\right)
$$

where $\sum_{j=1}^{N_{m}} \tilde{\lambda}_{j}=1, \tilde{\lambda}_{j} \geq 0$ for $j=1, \ldots, N_{m}$, and $\tilde{\mathbf{s}}_{1}, \ldots, \tilde{\mathbf{s}}_{N_{m}}$ are known joint signal constellations. Then, the approximate version of (12)-(13) can be formulated as follows:

$$
\begin{aligned}
\min _{\tilde{\boldsymbol{\lambda}}} & \max _{k \in\{1, \ldots, K\}} \tilde{\boldsymbol{\lambda}}^{T} \mathbf{g}_{k} \\
& \text { subject to } \tilde{\boldsymbol{\lambda}}^{T} \mathbf{h} \leq A, \quad \tilde{\boldsymbol{\lambda}}^{T} \mathbf{1}=1, \quad \tilde{\boldsymbol{\lambda}} \succeq \mathbf{0},
\end{aligned}
$$

where $\tilde{\boldsymbol{\lambda}} \triangleq\left[\tilde{\lambda}_{1} \cdots \tilde{\lambda}_{N_{m}}\right], \mathbf{g}_{k} \triangleq\left[G_{k}\left(\tilde{\mathbf{s}}_{1}\right) \cdots G_{k}\left(\tilde{\mathbf{s}}_{N_{m}}\right)\right], \mathbf{h} \triangleq$ $\left[H\left(\tilde{\mathbf{s}}_{1}\right) \cdots H\left(\tilde{\mathbf{s}}_{N_{m}}\right)\right], ' \succeq$ ' represents the componentwise larger than or equal to sign, and $\mathbf{0}$ and $\mathbf{1}$ denote vectors of zeros and ones, respectively. In other words, instead of considering all possible PDFs as in (15)-(16), a number of known joint signal constellations are considered, and the optimal weights, $\tilde{\lambda}$, corresponding to those joint signal constellations are searched for. In general, the solution of (18)-(19) provides an approximation to the optimal solution that is obtained from (15)-(16). The approximation accuracy can be improved by increasing $N_{m}$, i.e., by considering a larger number of elements in the set of possible signal values, $\tilde{\mathbf{s}}_{1}, \ldots, \tilde{\mathbf{s}}_{N_{m}}$, in (17). In addition, if $\tilde{\mathbf{s}}_{1}, \ldots, \tilde{\mathbf{s}}_{N_{m}}$ contain all the possible joint signal constellations (e.g., for a digital system), then the solution of (18)-(19) becomes exact.

By defining an auxiliary variable $t$, an equivalent form of (18)-(19) can be obtained as follows:

$$
\begin{aligned}
\min _{t, \tilde{\boldsymbol{\lambda}}} & t \\
\text { subject to } & \tilde{\boldsymbol{\lambda}}^{T} \mathbf{g}_{k} \leq t, k=1, \ldots, K \\
& \tilde{\boldsymbol{\lambda}}^{T} \mathbf{h} \leq A, \quad \tilde{\boldsymbol{\lambda}}^{T} \mathbf{1}=1, \quad \tilde{\boldsymbol{\lambda}} \succeq \mathbf{0} .
\end{aligned}
$$

It is noted that (20)-(22) corresponds to linearly constrained linear programming $(L C L P)$. Therefore, it can be solved efficiently in polynomial time [39].

\section{Optimal Selection of Fixed Signal Constellations as a Spe- cial Case of Optimal Power Control with Signal Constellation Randomization}

Conventionally, a fixed signal constellation is employed for each user in a multiuser system [1], [8]. This conventional scenario can be considered as a special case of power control with signal constellation randomization in which the PDF of $\boldsymbol{S}$ in (5), $p_{\boldsymbol{S}}$, is modeled as $p_{\boldsymbol{S}}(\mathbf{x})=\delta(\mathbf{x}-\mathbf{s})$. Then, the optimization problem in (12)-(13) reduces to the optimal selection of fixed signal constellations problem, which is expressed as

$$
\min _{\mathbf{s}} \max _{k \in\{1, \ldots, K\}} G_{k}(\mathbf{s}) \text { subject to } H(\mathbf{s}) \leq A .
$$

In other words, the optimal fixed signal constellations that minimize the maximum probability of error are obtained under the average power constraint. As investigated in Section V, the optimal fixed signal constellations approach can result in degraded performance in certain scenarios compared to the optimal power control with signal constellation randomization However, it has lower computational complexity, which can be desirable in certain applications.

\section{Special Case: Sign Detectors}

In this section, optimal power control with signal constellation randomization is studied in detail for symmetric signaling when sign detectors are employed at the receivers. In addition to the statistical characterization of the optimal solution, performance improvements that can be achieved via constellation randomization are quantified for interference limited scenarios.

Although sign detectors may not be optimal in the presence of interference [40], they facilitate simple implementation as they have low complexity and do not need any prior information about the interference. The use of sign detectors is justified also by the zero mean nature of the noise and interference (see (3)). It should be noted that the interference has zero mean since symmetric signaling and equally likely information bits are assumed. For these reasons, sign detectors are employed in many binary communications systems, such as in various wireless sensor network applications due to their low complexity and practicality [41].

For sign detectors, the decision rules at the receivers (see (4)) become

$$
\hat{i}_{k}=\phi_{k}\left(y_{k}\right)= \begin{cases}0, & y_{k}<0 \\ 1, & y_{k}>0\end{cases}
$$

for $k=1, \ldots, K$. In the case of $y_{k}=0$, the detector decides for bit 0 or bit 1 with equal probabilities. Then, for symmetric signaling (i.e., $S_{k}^{(1)}=-S_{k}^{(0)}$ for $\left.k=1, \ldots, K\right), G_{k}(\boldsymbol{S})$ in (11) can be expressed, after some manipulation, as

$$
G_{k}(\boldsymbol{S})=\frac{1}{2^{K-1}} \sum_{\mathbf{i}_{k} \in\{0,1\}^{K-1}} Q\left(\frac{S_{k}^{(1)}+\sum_{l=1, l \neq k}^{K} \rho_{k, l} S_{l}^{\left(i_{l}\right)}}{\sigma_{k}}\right) .
$$

In order to provide intuitions about the performance of constellation randomization in MAI limited scenarios, an asymptotical analysis is performed as $\sigma_{k} \rightarrow 0$ for $i=1, \ldots, K$. In this case, $G_{k}(\boldsymbol{S})$ in (25) can be expressed as

$$
G_{k}(\boldsymbol{S})=\frac{1}{2^{K-1}} \sum_{\mathbf{i}_{k} \in\{0,1\}^{K-1}} u\left(-S_{k}^{(1)}-\sum_{l=1, l \neq k}^{K} \rho_{k, l} S_{l}^{\left(i_{l}\right)}\right)
$$

where $u(\cdot)$ represents the unit step function defined as $u(x)=$ 1 for $x>0, u(x)=0.5$ for $x=0$ and $u(x)=0$ for $x<0$.

First, the following corollary to Proposition 1 is presented related to the probability distribution of the optimal joint signal constellation when sign detectors are employed.

Corollary 1: Assume that signal amplitudes take values from finite closed intervals, and $\sigma_{k} \rightarrow 0$ for $k=1, \ldots, K$. Then, an optimal solution to (12)-(13) can be expressed, for sign detectors and symmetric signaling, as

$$
p_{\boldsymbol{S}}(\mathbf{s})=\sum_{j=1}^{K} \lambda_{j} \delta\left(\mathbf{s}-\mathbf{s}_{j}\right)
$$


where $\sum_{j=1}^{K} \lambda_{j}=1$ and $\lambda_{j} \geq 0$ for $j=1, \ldots, K$.

Proof: Please see Appendix C.

In other words, instead of the generic solution in (14), which specifies a randomization among up to $(K+1)$ different signal constellations for each user, a randomization among up to $K$ different signal constellations is sufficient in this scenario. This is mainly due to the fact that, as $\sigma_{k} \rightarrow 0$ for $k=1, \ldots, K$, $G_{k}(S)$ in (26) depends only on the relative signal amplitudes, which makes the average power constraint in (13) ineffective (i.e., signal amplitudes can be scaled by the same positive number without affecting $G_{k}(\boldsymbol{S})$ 's and $H(\boldsymbol{S})$ in (9) can be adjusted appropriately).

Next, the aim is to compare the performance of the power control with signal constellation randomization and fixed signal constellations approaches for sign detectors in the absence of noise. Assume without loss of generality that $S_{k}^{(1)}$ 's are positive. Then, it is observed that both approaches can achieve zero probability of error if there exists a joint signal constellation $S$ such that $^{7}$

$$
S_{k}^{(1)}>\sum_{l=1, l \neq k}^{K}\left|\rho_{k, l}\right| S_{l}^{(1)}, \quad \forall k \in\{1, \ldots, K\} .
$$

This simple condition follows from (26) since it guarantees that the argument of the unit step function is negative for all bit combinations (recalling that $S_{l}^{(0)}=-S_{l}^{(1)}$ as symmetric signaling is considered). This is similar to the no error floor condition in classical multiuser systems [1]. (However, we still state it explicitly in order to employ it in Proposition 2 and Proposition 3 below.)

The condition in (28) corresponds to scenarios in which MAI is not significant and no error floor occurs due to interference. However, this condition may not be satisfied in certain cases and the MAI can be significant. For those cases, it is important to quantify the maximum amount of improvement (in terms of the minimum value of the maximum probability of error) that can be achieved via the power control with signal constellation randomization approach over the fixed signal constellations approach. Let $\mathrm{P}_{\text {rnd }}$ denote the minimum value of the maximum probability of error corresponding to the optimal power control with signal constellation randomization, which is obtained as the solution of (12)-(13). In addition, let $\mathrm{P}_{\text {fix }}$ denote the minimum value of the maximum probability of error for the optimal fixed signal constellations approach, which is obtained from (23). Then, the following proposition specifies the maximum asymptotical improvement due to signal constellation randomization.

Proposition 2: Suppose there exist no signal amplitudes that satisfy (28). Then, for sign detectors and symmetric signaling, the maximum asymptotical improvement ratio is equal to the number of users. In other words,

$$
1 \leq \lim _{\sigma_{1}, \ldots, \sigma_{K} \rightarrow 0} \frac{\mathrm{P}_{\text {fix }}}{\mathrm{P}_{\text {rnd }}} \leq K .
$$

Also, the maximum asymptotical improvement ratio, $K$, is

\footnotetext{
${ }^{7}$ It can be assumed without loss of generality that $S$ satisfies the power constraint in (13) since scaling the joint signal constellation $\boldsymbol{S}$ by any positive number does not affect the inequalities in (28).
}

achieved if there exist signal amplitudes such that

$$
S_{k}^{(1)}>\sum_{l=1, l \neq k}^{K}\left|\rho_{k, l}\right| S_{l}^{(1)}, \forall k \in\{1, \ldots, K\} \backslash\left\{k^{*}\right\}
$$

and

$$
\begin{aligned}
-2 \min _{l \in\{1, \ldots, K\} \backslash\left\{k^{*}\right\}} & \left\{\left|\rho_{k^{*}, l}\right| S_{l}^{(1)}\right\} \\
& <S_{k^{*}}^{(1)}-\sum_{l=1, l \neq k^{*}}^{K}\left|\rho_{k^{*}, l}\right| S_{l}^{(1)}<0
\end{aligned}
$$

for any $k^{*} \in\{1, \ldots, K\}$.

Proof: In order to prove the inequality in (29), it is first observed that $\mathrm{P}_{\text {fix }} / \mathrm{P}_{\text {rnd }} \geq 1$ is satisfied in all cases (even for finite $\sigma_{k}$ 's) since the fixed signal constellations approach is a special case of the power control with signal constellation randomization approach, as discussed in Section III-C. To prove the upper bound in (29), consider the case in which there exist signal amplitudes that satisfy the conditions in (30)-(31).

For fixed signal constellations, the average probability of error for user $k$ is given by $\mathrm{P}_{k}=G_{k}(\mathbf{s})$ for $k=1, \ldots, K$ (see (10)). Let $\mathbf{s}_{k^{*}}^{(1)}$ denote a joint signal constellation that satisfies the conditions in (30)-(31) for $k^{*} \in\{1, \ldots, K\}$. Based on the expression for $G_{k}$ in (26), it is obtained that $G_{k}\left(\mathbf{s}_{k^{*}}^{(1)}\right)=0$, $\forall k \in\{1, \ldots, K\} \backslash\left\{k^{*}\right\}$ since the argument of the unit step function, $-S_{k}^{(1)}-\sum_{l=1, l \neq k}^{K} \rho_{k, l} S_{l}^{\left(i_{l}\right)}$, is always negative due to the conditions in (30). ${ }^{8}$ On the other hand, the value of $G_{k^{*}}\left(\mathbf{s}_{k^{*}}^{(1)}\right)$ is obtained as follows. The condition in (31) can be expressed as

$$
\begin{aligned}
\sum_{l=1, l \neq k^{*}}^{K}\left|\rho_{k^{*}, l}\right| S_{l}^{\left(i_{l}\right)} & -2 \min _{l \in\{1, \ldots, K\} \backslash\left\{k^{*}\right\}}\left\{\left|\rho_{k^{*}, l}\right| S_{l}^{(1)}\right\} \\
& <S_{k^{*}}^{(1)}<\sum_{l=1, l \neq k^{*}}^{K}\left|\rho_{k^{*}, l}\right| S_{l}^{\left(i_{l}\right)} .
\end{aligned}
$$

Due to symmetric signaling, $\quad \sum_{l=1, l \neq k^{*}}^{K}\left|\rho_{k^{*}, l}\right| S_{l}^{\left(i_{l}\right)}$ corresponds to the maximum value of $-\sum_{l=1, l \neq k^{*}}^{K} \rho_{k^{*}, l} S_{l}^{\left(i_{l}\right)}$ for $\mathbf{i}_{k^{*}} \in\{0,1\}^{K-1} \quad$ (see (26)). Similarly, $\sum_{l=1, l \neq k^{*}}^{K}\left|\rho_{k^{*}, l}\right| S_{l}^{\left(i_{l}\right)}-2_{l \in\{1, \ldots, K\} \backslash\left\{k^{*}\right\}}\left\{\left|\rho_{k^{*}, l}\right| S_{l}^{(1)}\right\}$ is equal to the second largest value of $-\sum_{l=1, l \neq k^{*}}^{K} \rho_{k^{*}, l} S_{l}^{\left(i_{l}\right)}$ since that value is achieved when all the $-\rho_{k^{*}, l} S_{l}^{\left(i_{l}\right)}$ terms are taken to be positive except for the one with the smallest absolute value. Therefore, under the condition in (32), $S_{k^{*}}^{(1)}$ is between the maximum and the second largest value of $-\sum_{l=1, l \neq k^{*}}^{K} \rho_{k^{*}, l} S_{l}^{\left(i_{l}\right)}$, which implies that the argument of the unit step function in (26), $-S_{k^{*}}^{(1)}-\sum_{l=1, l \neq k^{*}}^{K} \rho_{k^{*}, l} S_{l}^{\left(i_{l}\right)}$, is negative for all possible signal combinations except for one of them. Hence, the unit step function in (26) becomes zero for $\left(2^{K-1}-1\right)$ combinations and becomes one only for one combination, which results in $G_{k^{*}}\left(\mathbf{s}_{k^{*}}^{(1)}\right)=1 / 2^{K-1}$. Overall, the maximum value of the average probability of error is given by $\max _{k} \mathrm{P}_{k}=\max _{k} G_{k}\left(\mathbf{s}_{k^{*}}^{(1)}\right)=1 / 2^{K-1}$ for the fixed signal constellations approach when a joint signal

\footnotetext{
${ }^{8}$ It is recalled that $S_{l}^{(1)}$,s are assumed to be positive without loss of generality and $S_{l}^{(0)}=-S_{l}^{(1)}$ due to symmetric signaling.
} 
constellation that satisfies the conditions in (30)-(31) is employed. Since it is impossible to set all $G_{k}$ 's to zero simultaneously due to the assumption in the proposition, $1 / 2^{K-1}$ presents the minimum value for the maximum average probability of error. Therefore, the solution of (23) is given by $\mathrm{P}_{\text {fix }}=1 / 2^{K-1}$ under the conditions in (30)-(31).

For the power control with signal constellation randomization approach, the average probability of error for user $k$ is given by $\mathrm{P}_{k}=\mathrm{E}\left\{G_{k}(\boldsymbol{S})\right\}$ for $k=1, \ldots, K$ (see (10)). Due to the assumption in the proposition, there does not exist any signal amplitudes that set all $G_{k}$ 's to zero simultaneously. Therefore, it is impossible to set all the $\mathrm{P}_{k}$ values to zero even in the signal constellation randomization approach. However, signal constellation randomization can be used to reduce the maximum average probability of error by means of randomization/time-sharing. To explain this point, consider joint signal constellations $\mathbf{s}_{k^{*}}^{(1)}$ that satisfy the conditions in (30)-(31). As discussed in the previous paragraph, these vectors result in $G_{k}\left(\mathbf{s}_{k^{*}}^{(1)}\right)=0, \forall k \in\{1, \ldots, K\} \backslash\left\{k^{*}\right\}$ and $G_{k^{*}}\left(\mathbf{s}_{k^{*}}^{(1)}\right)=1 / 2^{K-1}$ for $k^{*} \in\{1, \ldots, K\}$. Since the aim is to minimize $\max _{k} \mathrm{E}\left\{G_{k}(\boldsymbol{S})\right\}$ over all possible PDFs for the joint signal constellation, the optimal solution is obtained by an equalizer rule [32], which sets $\mathrm{E}\left\{G_{1}(\boldsymbol{S})\right\}=\mathrm{E}\left\{G_{2}(\boldsymbol{S})\right\}=$ $\cdots=\mathrm{E}\left\{G_{K}(\boldsymbol{S})\right\}$. For this equalizer rule, the optimal PDF for the joint signal constellation can be expressed as

$$
p_{\boldsymbol{S}}(\mathbf{s})=\frac{1}{K} \sum_{k^{*}=1}^{K} \delta\left(\mathbf{s}-\mathbf{s}_{k^{*}}^{(1)}\right) .
$$

Therefore,

$$
\mathrm{E}\left\{G_{k}(\boldsymbol{S})\right\}=\frac{1}{K} \sum_{k^{*}=1}^{K} G_{k}\left(\mathbf{s}_{k^{*}}^{(1)}\right)=\frac{1}{K 2^{K-1}}
$$

is obtained for all $k \in\{1, \ldots, K\}$. Hence, $\max _{k} \mathrm{P}_{k}=$ $\max _{k} \mathrm{E}\left\{G_{k}(\boldsymbol{S})\right\}=1 /\left(K 2^{K-1}\right)$. Since it is impossible to set all $G_{k}(\mathbf{s})$ 's to zero for a given $\mathbf{s}$ due to the assumption in the proposition and setting $(K-1)$ of them to zero and one of them to $1 / 2^{K-1}$ corresponds to the optimal scenario for a given $\mathbf{s}$, the solution in (33) presents the optimal solution of $\min _{p_{S}} \max _{k} \mathrm{P}_{k}$, which is equal to $1 /\left(K 2^{K-1}\right)$. Hence, $\mathrm{P}_{\text {rnd }}=1 /\left(K 2^{K-1}\right)$ is obtained.

Overall, an improvement ratio of $\mathrm{P}_{\text {fix }} / \mathrm{P}_{\text {rnd }}=$ $K 2^{K-1} / 2^{K-1}=K$ is achieved under the conditions in the proposition. Finally, it is shown that $K$ presents an upper limit on the asymptotical improvement ratio for the scenario in the proposition. To that aim, let the probability distribution of the joint signal constellation corresponding to the optimal power control with signal constellation randomization approach be expressed as in (27). Then, the minimum value of the maximum probability of error in the power control with signal constellation randomization approach is given by $\mathrm{P}_{\mathrm{rnd}}=\max _{k} \sum_{j=1}^{K} \lambda_{j} G_{k}\left(\mathbf{s}_{j}\right)$, where $\sum_{j=1}^{K} \lambda_{j}=1$. Next, the following inequalities are obtained:

$$
\mathrm{P}_{\mathrm{rnd}}=\max _{k} \sum_{j=1}^{K} \lambda_{j} G_{k}\left(\mathbf{s}_{j}\right) \geq \frac{1}{K} \sum_{k=1}^{K} \sum_{j=1}^{K} \lambda_{j} G_{k}\left(\mathbf{s}_{j}\right)
$$

$$
\begin{aligned}
& \geq \frac{1}{K} \sum_{j=1}^{K} \lambda_{j}\left(\min _{\mathbf{s}} \sum_{k=1}^{K} G_{k}(\mathbf{s})\right)=\frac{1}{K} \min _{\mathbf{s}} \sum_{k=1}^{K} G_{k}(\mathbf{s}) \\
& \geq \frac{1}{K} \min _{\mathbf{s}} \max _{k} G_{k}(\mathbf{s})=\frac{1}{K} \mathrm{P}_{\text {fix }}
\end{aligned}
$$

The inequalities in (35) and (37) follow from the fact that $K \max _{k} y_{k} \geq \sum_{k=1}^{K} y_{k} \geq \max _{k} y_{k}$ for $y_{k} \geq 0 \forall k$, and the inequality in (36) is obtained by performing an additional minimum operation. Based on (35)-(37), $\mathrm{P}_{\text {fix }} / \mathrm{P}_{\text {rnd }} \leq K$ is obtained.

Proposition 2 states that in interference-limited scenarios, the maximum average probability of error can be reduced by a factor of up to $K$ via signal constellation randomization. This improvement ratio is related to the result in Corollary 1 , which states that a randomization among up to $K$ joint signal constellations can be employed to reduce the maximum average probability of error compared to the fixed signal constellations case. By employing randomization among multiple different joint signal constellations, the average probabilities of error for different users can be equalized to a certain extent, which can reduce the maximum value of the average probabilities of error. In practice, the randomization operation can be implemented in the time domain via time-sharing (or in the frequency domain for multichannel systems) by employing each joint signal constellation for a certain fraction of time.

In Proposition 2, the upper and lower bounds on the asymptotical improvements that can be achieved via signal constellation randomization are presented, and the conditions under which the upper bound is achieved are specified. In the following proposition, conditions are obtained to specify when the lower bound in (29) is achieved; that is, when the use of signal constellation randomization does not provide any performance improvements over the use of fixed signal constellations.

Proposition 3: Consider sign detectors and symmetric signaling, and assume that there exist no signal amplitudes that satisfy (28). In addition, define $\mathbf{s}^{*}$ as a joint signal constellation that minimizes the sum of the average error probabilities of the users. Then, if $G_{1}\left(\mathbf{s}^{*}\right)=G_{2}\left(\mathbf{s}^{*}\right)=\cdots=G_{K}\left(\mathbf{s}^{*}\right)$, $\mathrm{s}^{*}$ is a solution of the optimal power control with signal constellation randomization problem, and the asymptotical improvement ratio is equal to one; that is,

$$
\lim _{\sigma_{1}, \ldots, \sigma_{K} \rightarrow 0} \frac{\mathrm{P}_{\text {fix }}}{\mathrm{P}_{\text {rnd }}}=1 .
$$

Proof: The joint signal constellation $\mathrm{s}^{*}$ defined in the proposition can be expressed as

$$
\mathbf{s}^{*}=\arg \min _{\mathbf{s}} \sum_{k=1}^{K} G_{k}(\mathbf{s}) .
$$

Also, by definition, $\mathrm{P}_{\text {fix }}=\min _{\mathbf{s}} \max _{k} G_{k}(\mathbf{s})$, which can be bounded from below as follows:

$$
\mathrm{P}_{\mathrm{fix}}=\min _{\mathbf{s}} \max _{k} G_{k}(\mathbf{s}) \geq \frac{1}{K} \min _{\mathbf{s}} \sum_{k=1}^{K} G_{k}(\mathbf{s})=G_{1}\left(\mathbf{s}^{*}\right)
$$

where the condition in the proposition, $G_{1}\left(\mathbf{s}^{*}\right)=G_{2}\left(\mathbf{s}^{*}\right)=$ $\cdots=G_{K}\left(\mathbf{s}^{*}\right)$, is used to obtain the last equality in (39). Since $\min _{\mathbf{s}} \max _{k} G_{k}(\mathbf{s})$ is lower bounded by $G_{1}\left(\mathbf{s}^{*}\right)$ as stated 
in (39) and this lower bound can be achieved for $\mathbf{s}=\mathbf{s}^{*}$, $\mathrm{P}_{\mathrm{fix}}=G_{1}\left(\mathbf{s}^{*}\right)$ is obtained. Therefore, $\mathbf{s}^{*}$ is a solution for the optimal selection of fixed signal constellations problem, as claimed in the proposition. In addition, from (35) and (36), $\mathrm{P}_{\text {rnd }} \geq \frac{1}{K} \min _{\mathrm{s}} \sum_{k=1}^{K} G_{k}(\mathrm{~s})$, which becomes $\mathrm{P}_{\text {rnd }} \geq$ $G_{1}\left(\mathbf{s}^{*}\right)=\mathrm{P}_{\text {fix }}$ under the conditions in the proposition. Since $\mathrm{P}_{\text {rnd }} \leq \mathrm{P}_{\text {fix }}$ is also satisfied by definition (as the fixed signal constellations approach is a special case of power control with signal constellation randomization), $\mathrm{P}_{\text {rnd }}=\mathrm{P}_{\text {fix }}$ is obtained.

Proposition 3 implies that if a joint signal constellation that minimizes the sum of the average error probabilities of the users also equalizes the average error probabilities of the users, then it is a solution of both the optimal selection of fixed signal constellations and the optimal power control with signal constellation randomization problems for the scenario in the proposition. In other words, the signal constellation randomization approach cannot provide any performance improvements over the fixed signal constellations approach, and the two approaches yield the same solution, namely, $p_{\mathbf{s}}(\mathbf{s})=\delta\left(\mathbf{s}-\mathbf{s}^{*}\right)$.

\section{Performance Evaluation}

In this section, simulations are performed in order to compare the performance of the power control with signal constellation randomization approach against various approaches that employ fixed signal constellations. Namely, the following techniques are investigated in the simulations.

Power Control with Signal Constellation Randomization: Randomization of signal constellations is performed in an optimal or suboptimal manner based on the formulations in (15)-(16) or (20)-(22), respectively. In the following, the former approach is called optimal randomization of signal constellations, whereas the latter is named constellation randomization with relaxation. Optimal randomization of signal constellations can have prohibitive computational complexity when the number of users is high. Therefore, constellation randomization with relaxation is employed for large numbers of users in order to reduce the computational complexity.

Optimal Fixed Signal Constellations: In this case, fixed signal constellations are considered for all users, and the optimal solution is obtained from (23), as discussed in Section III-C.

Fixed Signal Constellations at Power Limit: Instead of obtaining the optimal fixed signal constellations from (23), one can also consider a fixed signal constellations scheme that equalizes signal-to-interference-plus-noise ratios (SINRs) at different receivers, and utilizes all the available power at the transmitter [9]. The SINR at the receiver of user $k$ is calculated from (3) as $\operatorname{SINR}_{k}=\mathrm{E}\left\{\left|S_{k}^{\left(i_{k}\right)}\right|^{2}\right\} /\left(\mathrm{E}\left\{\left|\sum_{l \neq k} \rho_{k, l} S_{l}^{\left(i_{l}\right)}\right|^{2}\right\}+\right.$ $\left.\sigma_{k}^{2}\right)$, which becomes $\operatorname{SINR}_{k}=\left|S_{k}^{(1)}\right|^{2} /\left(\sum_{l \neq k} \rho_{k, l}^{2}\left|S_{l}^{(1)}\right|^{2}+\right.$ $\left.\sigma_{k}^{2}\right)$ for symmetric signaling and fixed signal constellations. In the fixed signal constellations at the power limit approach, $S_{1}^{(1)}, \ldots, S_{k}^{(K)}$ are chosen such that $\operatorname{SINR}_{1}=\cdots=\operatorname{SINR}_{K}$ and $\sum_{k=1}^{K}\left|S_{k}^{(1)}\right|^{2}=A$. Although this approach can provide very low complexity solutions, its performance is inferior to both the optimal fixed signal constellations and optimal

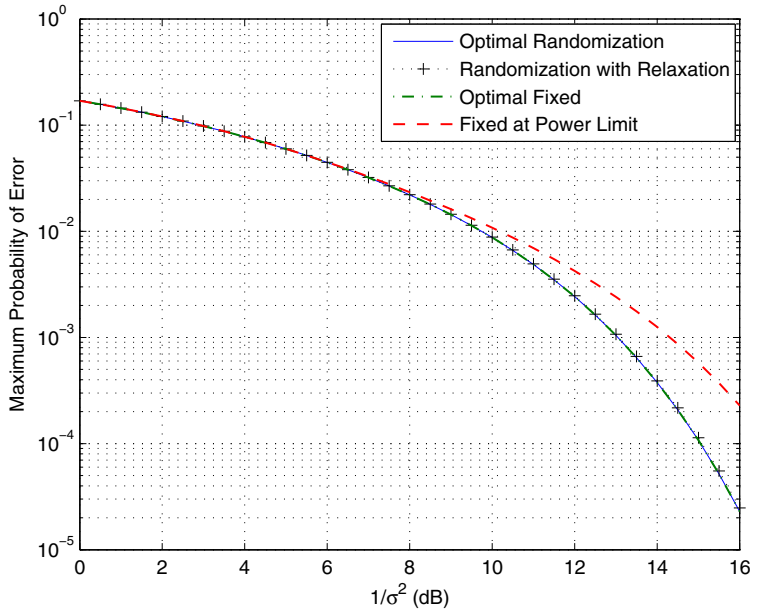

Fig. 2. Maximum probabilities of error versus $1 / \sigma^{2}$ for the optimal randomization of signal constellations ("Optimal Randomization"), constellation randomization with relaxation ("Randomization with Relaxation"), optimal fixed signal constellations ("Optimal Fixed"), and fixed signal constellations at the power limit ("Fixed at Power Limit") approaches, where $K=3$, $\rho_{1,2}=0.1, \rho_{1,3}=0.2, \rho_{2,3}=0.3$, and $A=3$.

randomization of signal constellations approaches in general, as investigated below.

In the simulations, equally likely information bits are assumed, and symmetric signaling is considered. Also, the users employ sign detectors at the receivers, and the standard deviations of the noise at the receivers are taken to be equal, that is, $\sigma_{k}=\sigma, k=1, \ldots, K$. In addition, as stated after (3), $\rho_{k, l}$ 's are set to one for $k=l$; that is, $\rho_{k, k}=1$ for $k=1, \ldots, K$.

First, a 3-user scenario is considered, that is, $K=3$, and the crosscorrelations between the pseudo-noise signals for different users are set to $\rho_{1,2}=0.1, \rho_{1,3}=0.2$, and $\rho_{2,3}=0.3$. Also, the average power constraint $A$ in (7) is taken as 3. In Fig. 2, the maximum probabilities of error are plotted versus $1 / \sigma^{2}$ for the optimal randomization of signal constellations, constellation randomization with relaxation, optimal fixed signal constellations, and fixed signal constellations at the power limit approaches. For the optimal randomization of signal constellations approach, the PSO algorithm is employed with 2000 iterations and 50 particles in order to obtain the solution of (15)-(16) (please refer to [42] for details of the PSO algorithms). For the constellation randomization with relaxation approach, the possible signal values for bit 1 are selected as 32 different amplitudes equally spaced between 0 and 1.4, and the negatives of these possible values are employed for bit 0 . From the figure, it is observed that the optimal randomization of signal constellations, the constellation randomization with relaxation, and the optimal fixed signal constellations approaches have almost the same performance, and the fixed signal constellations at the power limit approach has higher maximum error probabilities for small values of $\sigma^{2}$, i.e., for low noise powers. On the other hand, all the approaches have similar performance in the noise limited scenarios. It is concluded that it is not optimal in general to employ fixed signal constellations that equate the 


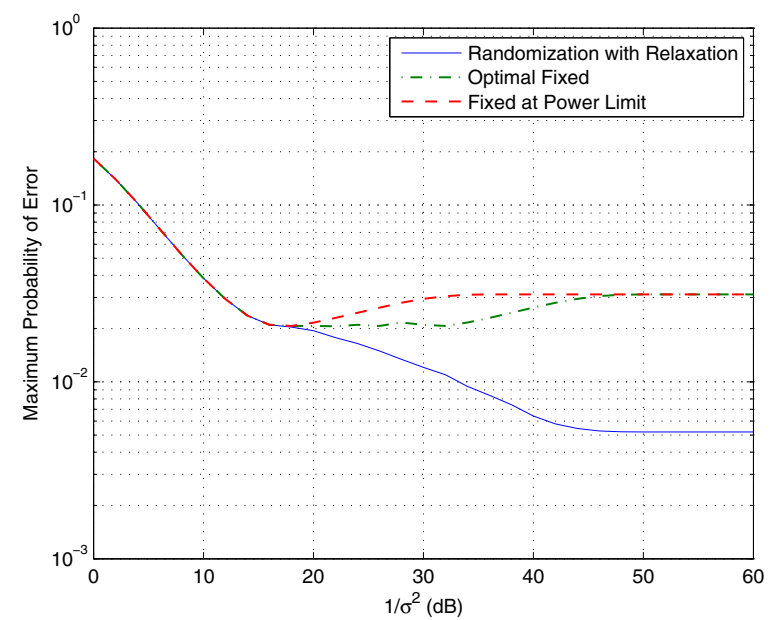

Fig. 3. Maximum probabilities of error versus $1 / \sigma^{2}$ for the constellation randomization with relaxation, optimal fixed signal constellations, and fixed signal constellations at the power limit approaches, where $K=6, \rho_{k, l}=$ 0.21 for all $k \neq l$, and $A=6$.

\section{SINRs of different users.}

Next, a 6-user scenario is considered, that is, $K=6$, and the crosscorrelations between the pseudo-noise signals for different users are set to 0.21 ; i.e., $\rho_{k, l}=0.21$ for $k \neq l$. Also, the average power constraint $A$ in (7) is taken as 6 . In Fig. 3, the maximum probabilities of error are illustrated for the constellation randomization with relaxation, optimal fixed signal constellations, and fixed signal constellations at the power limit approaches. Since the solution of (15)-(16) requires a search over a $(K+1)^{2}=49$ dimensional space, global optimization techniques may not be employed to obtain the optimal randomization of signal constellations solution in this scenario. Therefore, randomization of signal constellations is performed only via the constellation randomization with relaxation approach, which is based on (20)-(22). In obtaining the solution for this approach, the signal amplitude for information bit 1 of each user is modeled to take values from 0 to 1.4 with an increment of $0.2 .{ }^{9}$ Then, the optimal weights for these possible signal amplitudes are obtained from (20)-(22) via CVX: Matlab Software for Disciplined Convex Programming [43]. The use of a finite set of signal amplitudes can be justified by considering a digital system in which a number of bits are used to represent each signal amplitude. In this scenario, a 4-bit representation is considered as there are 8 possible signal values, $\{0,0.2,0.4,0.6,0.8,1,1.2,1.4\}$, for information bit 1 and the negative of these values for information bit 0. From Fig. 3, it is observed that the constellation randomization with relaxation approach outperforms the approaches that employ fixed signal constellations for small noise variances; that is, for MAI limited scenarios. In addition, the optimal fixed signal constellations approach achieves lower maximum probabilities of error than the fixed signal constellations at the power limit approach for medium

\footnotetext{
${ }^{9}$ Since symmetric signaling is considered, the possible signal amplitudes for bit 0 are from -1.4 to 0 with an increment of 0.2 .
}

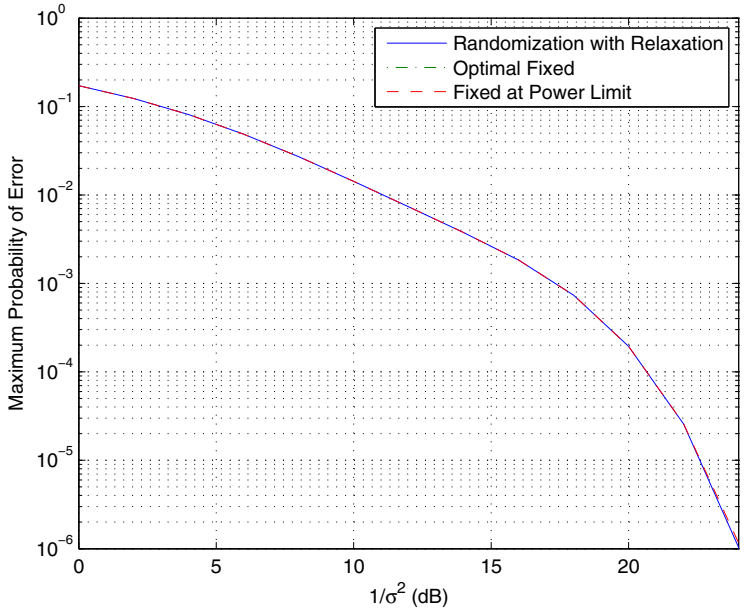

Fig. 4. Maximum probabilities of error versus $1 / \sigma^{2}$ for the constellation randomization with relaxation, optimal fixed signal constellations, and fixed signal constellations at the power limit approaches, where $K=6, \rho_{k, l}=$ 0.15 for all $k \neq l$, and $A=6$.

range of $\sigma$ values. ${ }^{10}$ Another important observation from the figure is that, for small values of $\sigma$, the constellation randomization approach achieves a 6 times improvement in the maximum probability of error compared to the optimal fixed signal constellations approach, as claimed in Proposition 2. In fact, it can be shown that the assumptions in the proposition are satisfied in this scenario. Namely, there exist no signal amplitudes that satisfy (28), and the conditions in (30)-(31) are satisfied, for example, when all $S_{k}^{(1)}$, s are 1.2 except for one of them, which is equal to 0.8 .

In addition, consider the same scenario as for Fig. 3, but assume that $\rho_{k, l}=0.15$ for $k \neq l$. In this case, the conditions in (28) are satisfied. Therefore, no error floors are expected and the MAI does not become a limiting factor. The error performances are illustrated in Fig. 4 for this scenario. It is observed that the maximum probabilities of error decrease towards zero as the noise variance is reduced, and all the algorithms have almost the same error performance. As another example, the results in Fig. 5 are presented when $\rho_{k, l}=0.25$ for $k \neq l$. In this case, since the crosscorrelation is high, the MAI is very effective and very high error probabilities are encountered. Also, it can be shown that the conditions in (28) and those in (30)-(31) are not satisfied for this scenario. In Fig. 5, the constellation randomization approach provides improvements over the approaches with fixed signal constellations, which have the same performance. However, the improvement ratio is smaller than 6 in this scenario, which is about 1.4 at low $\sigma$ values.

In Fig. 6, the error probabilities of the different approaches are plotted versus $\rho$, where $\rho_{k, l}=\rho$ for $k \neq l$. In addition, the other parameters are set to $A=6, K=6$, and $\sigma=10^{-3}$. It is observed that the constellation randomization approach

\footnotetext{
${ }^{10}$ It is also observed that the error probabilities of the approaches that employ fixed constellations can increase in some cases even when the noise variance decreases. This is mainly because of the multi-modal nature of the overall noise, which is the sum of zero-mean Gaussian background noise and MAI. Please see [16] for a detailed discussion.
} 


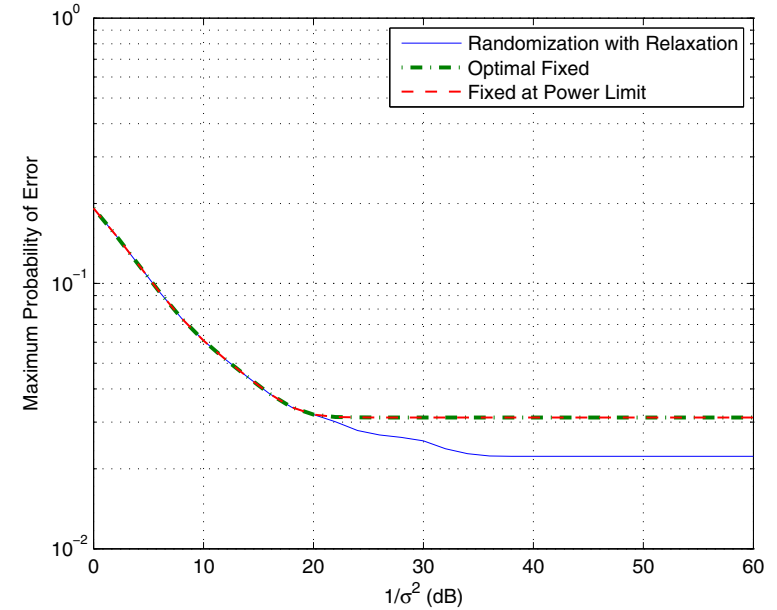

Fig. 5. Maximum probabilities of error versus $1 / \sigma^{2}$ for the constellation randomization with relaxation, optimal fixed signal constellations, and fixed signal constellations at the power limit approaches, where $K=6, \rho_{k, l}=$ 0.25 for all $k \neq l$, and $A=6$.

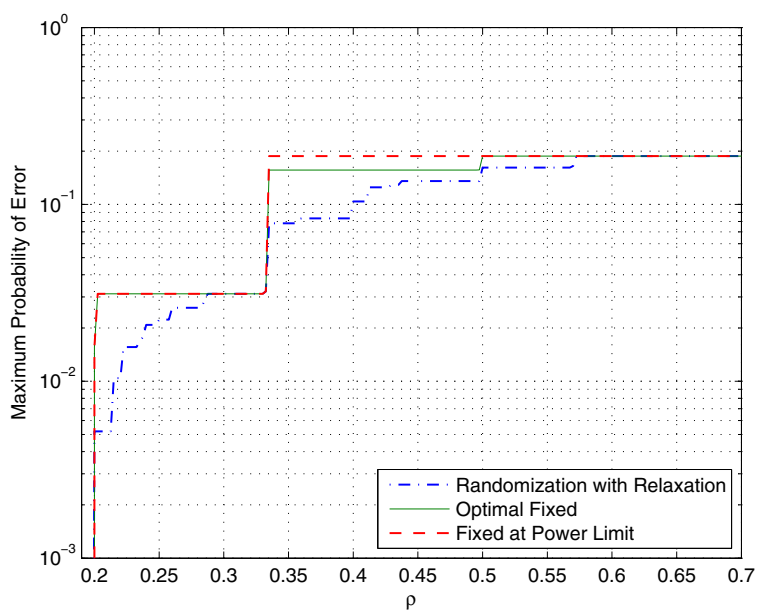

Fig. 6. Maximum probabilities of error versus $\rho$ for the constellation randomization with relaxation, optimal fixed signal constellations, and fixed signal constellations at the power limit approaches, where $K=6, A=6$, and $\sigma=10^{-3}$.

has lower error probabilities than the other approaches for $\rho \in[0.2,0.29]$ and $\rho \in[0.33,0.57]$. The improvement region and the amount of improvement depend on the relation among the system parameters. For example, as investigated in Section IV, an improvement ratio of $K$ is achieved for $\rho \in[0.2,0.215]$ (which can be obtained from the conditions in (30)-(31)), and lower improvement ratios are observed in other regions. Also, the optimal fixed signal constellations approach outperforms the fixed signal constellations at the power limit approach for certain range of $\rho$ values. However, it does not provide significant improvements in general.

In order to compare the error performance of the three approaches for different numbers of users, Fig. 7 is presented, where $A=6, \sigma=10^{-3}$, and $\rho_{k, l}=0.35$ for $k \neq l$. It is observed that the constellation randomization with relaxation approach provides improvements over the approaches that

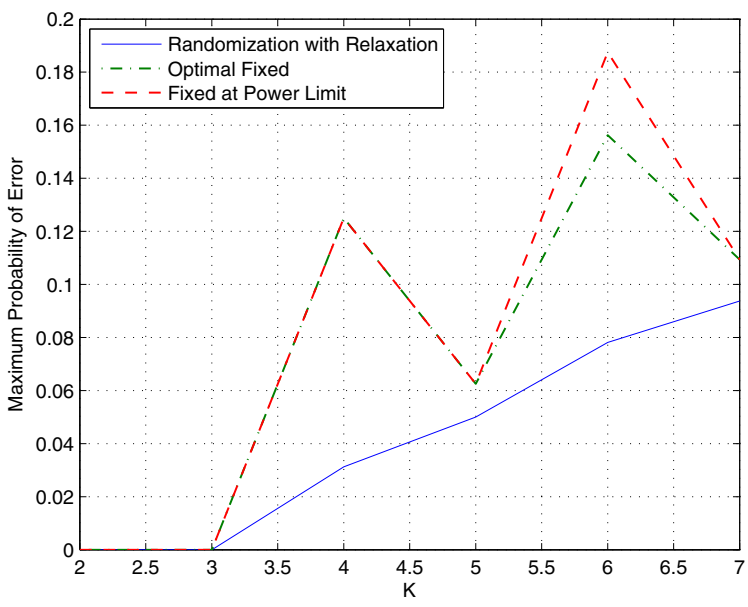

Fig. 7. Maximum probabilities of error versus the number of users, $K$, for the constellation randomization with relaxation, optimal fixed signal constellations, and fixed signal constellations at the power limit approaches, where $\sigma=10^{-3}, \rho_{k, l}=0.35$ for all $k \neq l$, and $A=6$.

employ fixed signal constellations when the number of users is larger than three, in which case the MAI becomes a dominating factor. Also, the approaches that employ fixed signal constellations achieve similar maximum probabilities of error in most cases. In addition, their error performance is observed to be a non-monotonic function of the number of users. For example, the errors are lower for $K=5$ than those for $K=4$. The reason for this seemingly counterintuitive behavior can be explained from the expression in (25), or more simply from (26) since $\sigma$ is sufficiently small. Considering the fixed signal constellations at the power limit approach, the signal amplitudes are set to $S_{k}^{(1)}=-S_{k}^{(0)}=\sqrt{A / K}$ for $k=1, \ldots, K$. Since $\rho_{k, l}=0.35$ for $k \neq l$, it can be shown for $K=4$ and $K=5$ that there is only one combination of the information bits of interfering users for which the argument of the unit step function in (26) becomes positive. Namely, when all the interfering signals are $-\sqrt{A / K}$, the argument of the unit step function becomes $-\sqrt{A / K}+0.35(K-1) \sqrt{A / K}$, which is positive for $K \geq 4$. On the other hand, when one of the interfering signals is set to $\sqrt{A / K}$, the argument becomes $-\sqrt{A / K}+0.35(K-3) \sqrt{A / K}$, which is negative for $K \leq 5$. (Of course, the result is still negative when more than one interfering signals are set to $\sqrt{A / K}$.) Therefore, for $K=4$ and $K=5, G_{k}(\boldsymbol{S})$ in (26) is equal to $1 / 2^{K-1}$ for $k=1, \ldots, K$ since the unit step function is 1 only for one combination and 0 otherwise. Hence, the maximum probability of error for $K=5$, is lower than that for $K=4$, as observed in Fig. 7. However, for $K=6$, there are multiple combinations of interfering signals for which the unit step function in (26) equal to one. Therefore, larger errors are observed in that case.

Finally, a scenario with $K=7$ users is considered, where $\rho_{k, l}=0.17$ for $k \neq l$, and $A=7$. In Fig. 8, the maximum probabilities of error are illustrated for the constellation randomization with relaxation, optimal fixed signal constellations, and fixed signal constellations at the power limit approaches. Similar observations to those for Fig. 3 can be made. In partic- 


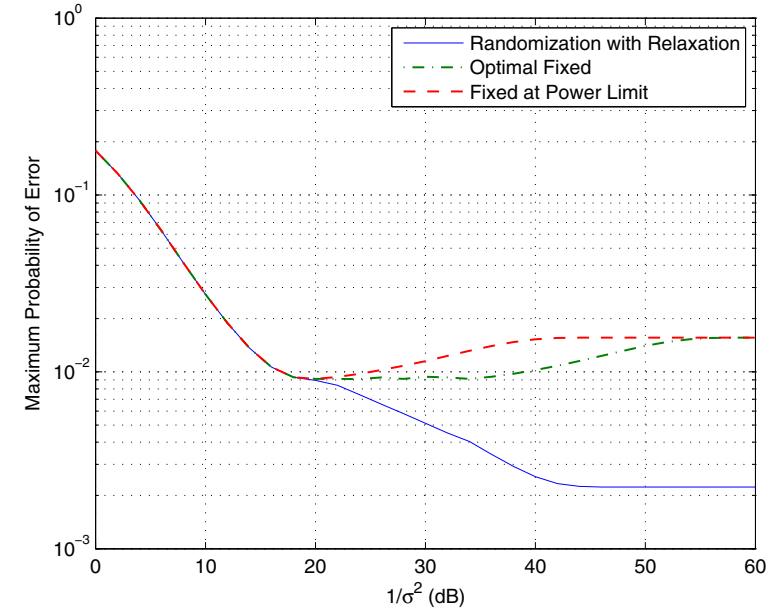

Fig. 8. Maximum probabilities of error versus $1 / \sigma^{2}$ for the constellation randomization with relaxation, optimal fixed signal constellations, and fixed signal constellations at the power limit approaches, where $K=7, \rho_{k, l}=$ 0.17 for all $k \neq l$, and $A=7$.

ular, it is observed that an improvement ratio of 7 is achieved at low noise variances; that is, the maximum probability of error is reduced by 7 times via the randomization of signal constellations, as claimed in Proposition 2.

The main observations from the simulation results can be summarized as follows: (i) Signal constellation randomization can provide performance improvements over the approaches that employ fixed signal constellations and the amount of improvement depends mainly on the noise level, the number of users, and the crosscorrelations between the pseudo-noise signals. (ii) The worst-case error rate of the optimal fixed signal constellations approach can be reduced by up to $K$ times via the optimal randomization of signal constellation approach. (iii) The fixed signal constellation approach that equalizes the SINRs of the users and utilizes all the available power has the worst performance among all the considered approaches.

\section{CONCLUDING REMARKS AND EXTENSIONS}

The optimal power control with signal constellation randomization has been proposed for the downlink of a multiuser DS-CDMA system. After presenting a formulation for the optimal power control with signal constellation randomization problem, it has been shown that an optimal joint signal constellation can be obtained by a randomization of $(K+1)$ or fewer distinct joint signal constellations, where $K$ denotes the number of users. In addition to the original nonconvex formulation, an approximate solution based on convex relaxation has been obtained. Then, detailed performance analysis has been performed when the receivers employ symmetric signaling and sign detectors. Specifically, the maximum asymptotical improvement ratio has been shown to be equal to the number of users, and the conditions under which the maximum and minimum asymptotical improvement ratios are achieved have been derived. Numerical examples have been presented to investigate the theoretical results.
Although the problem formulation is based on the minimax approach in (6), the results in this study can directly be extended to cover cases in which the users have different levels of importance. In that case, the expression in (6) can be replaced with $\min _{p_{S}} \max _{k \in\{1, \ldots, K\}} w_{k} \mathrm{P}_{k}$, where $w_{k}$ 's are non-negative weighting factors that are set according to the importance levels. Then, the definition of $G_{k}$ in (11) can be updated by multiplying the expression by $w_{k}$, and all the theoretical results in the remaining parts can be extended accordingly.

Finally, the theoretical approach employed for the binary multiuser systems in this work can also be utilized for $M$-ary systems with $M>2$. In that case, the definitions of the joint signal constellation in (5), and the auxiliary functions in (9) and (11) should be updated. Then, the results in Section III can be extended to $M$-ary systems as well.

\section{APPENDIX}

\section{A. Derivation of (10)}

For the generic decision rule in (4), the average probability of error for user $k$ can be expressed as $\mathrm{P}_{k}=0.5 \mathrm{P}\left\{Y_{k} \in\right.$ $\left.\Gamma_{k, 0} \mid i_{k}=1\right\}+0.5 \mathrm{P}\left\{Y_{k} \in \Gamma_{k, 1} \mid i_{k}=0\right\}$, which, based on (3), becomes

$$
\begin{aligned}
\mathrm{P}_{k} & =0.5 \mathrm{P}\left\{S_{k}^{(1)}+\sum_{\substack{l=1 \\
l \neq k}}^{K} \rho_{k, l} S_{l}^{\left(i_{l}\right)}+N_{k} \in \Gamma_{k, 0}\right\} \\
& +0.5 \mathrm{P}\left\{S_{k}^{(0)}+\sum_{\substack{l=1 \\
l \neq k}}^{K} \rho_{k, l} S_{l}^{\left(i_{l}\right)}+N_{k} \in \Gamma_{k, 1}\right\} .
\end{aligned}
$$

Since bits are equally likely, (40) can be expressed, by defining $\mathbf{i}_{k} \triangleq\left[\begin{array}{lll}i_{1} \cdots i_{k-1} & i_{k+1} \cdots i_{K}\end{array}\right]$, as

$$
\begin{array}{r}
\mathrm{P}_{k}=\frac{1}{2^{K}} \sum_{m \in\{0,1\}} \sum_{\mathbf{i}_{k} \in\{0,1\}^{K-1}} \mathrm{P}\left\{S_{k}^{(m)}+\sum_{\substack{l=1 \\
l \neq k}}^{K} \rho_{k, l} S_{l}^{\left(i_{l}\right)}\right. \\
\left.+N_{k} \in \Gamma_{k, 1-m}\right\}
\end{array}
$$

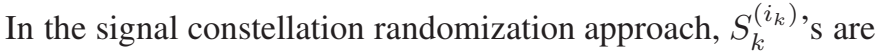
random variables. Hence, the probability expression in (41) can be calculated by first conditioning on given values of

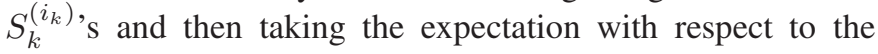
PDF of $S$; that is,

$$
\begin{aligned}
& \mathrm{P}\left\{S_{k}^{(m)}+\sum_{\substack{l=1 \\
l \neq k}}^{K} \rho_{k, l} S_{l}^{\left(i_{l}\right)}+N_{k} \in \Gamma_{k, 1-m}\right\}= \\
& \mathrm{E}\left\{\mathrm{P}\left\{N_{k}+S_{k}^{(m)}+\sum_{\substack{l=1 \\
l \neq k}}^{K} \rho_{k, l} S_{l}^{\left(i_{l}\right)} \in \Gamma_{k, 1-m} \mid \boldsymbol{S}\right\}\right\} .
\end{aligned}
$$

It is noted that the probability in (42) is calculated according to the PDF of $N_{k}$. By defining the expression inside the expectation in (42) as $G_{k}(\boldsymbol{S}),(10)$ and (11) are obtained from (41) and (42). 


\section{B. Proof of Proposition 1}

The proof can be obtained based on Carathéodory's theorem [44], [45] similarly to the proofs in [15], [19], [46]. First, define the following set: $U=\left\{\left(u_{0}, u_{1}, \ldots, u_{K}\right)\right.$ : $u_{0}=H(\mathbf{s}), u_{1}=G_{1}(\mathbf{s}), \ldots, u_{K}=G_{K}(\mathbf{s})$ for $\left.\mathbf{s} \in \mathcal{S}\right\}$, where $\mathcal{S} \triangleq\left[\mathrm{s}_{\min }, \mathrm{s}_{\max }\right]^{2 K}$, with $\mathrm{s}_{\min }$ and $\mathrm{s}_{\max }$ denoting the minimum and maximum signal amplitude values, respectively. Since the functions are continuous and $\mathcal{S}$ is a closed set, $U$ is closed and bounded; hence, it is a compact set. Therefore, the convex hull of $U$, denoted by $V$, is a closed subset of $\mathbb{R}^{K+1}$ [47]. Next, define set $W$ as follows: $W=\left\{\left(w_{0}, w_{1}, \ldots, w_{K}\right): w_{0}=\mathrm{E}\{H(\boldsymbol{S})\}, w_{1}=\right.$ $\left.\mathrm{E}\left\{G_{1}(\boldsymbol{S})\right\}, \ldots, w_{K}=\mathrm{E}\left\{G_{K}(\boldsymbol{S})\right\}, \forall p_{\boldsymbol{S}}(\mathbf{s}), \mathbf{s} \in \mathcal{S}\right\}$. Similar arguments as in [15], [19], [46], [48] can be used to conclude that set $W$ is equal to the convex hull of $U$; that is, $W=V$. Therefore, due to Carathéodory's theorem [44], [45], any point in $V$ (equivalently, in $W$ ) can be expressed as the convex combination of $(K+2)$ or fewer points in $U$ since the dimension of $U$ is smaller than or equal to $(K+1)$. Since the optimization problem in (12)-(13) aims to minimize the maximum of $\mathrm{E}\left\{G_{k}(\boldsymbol{S})\right\}$ 's, the optimal solution must correspond to the boundary of $W$. (Note that $W$ contains its boundary as it is a closed set.) Since any point at the boundary of $W$ can be expressed as the convex combination of at most $(K+1)$ elements in $U$ [44], an optimal PDF can be represented as in (14).

\section{Proof of Corollary 1}

As $\sigma_{k} \rightarrow 0$ for $k=1, \ldots, K, G_{k}(S)$ 's are expressed as in (26). Due to the unit step function in (26), scaling a joint signal constellation by a positive value does not affect the probabilities of error; that is, $G_{k}(\mathbf{s})=G_{k}(c \mathbf{s})$ for all $c>0$. Therefore, for each $\mathbf{s}$, there exists a positive constant $c$ for which $G_{k}$ 's are unchanged but $H(c \mathbf{s})=c^{2} H(\mathbf{s}) \leq A$ (see (9)). Hence, the average power constraint in (13) becomes ineffective in this scenario. Therefore, the proof of Proposition 1 in Appendix B can be applied in this case by redefining sets $U$ and $W$ as $U=\left\{\left(u_{0}, u_{1}, \ldots, u_{K-1}\right): u_{0}=G_{1}(\mathbf{s}), \ldots, u_{K-1}=\right.$ $G_{K}(\mathbf{s})$ for $\left.\mathbf{s} \in \mathcal{S}\right\}$ and $W=\left\{\left(w_{0}, w_{1}, \ldots, w_{K-1}\right)\right.$ : $\left.w_{0}=\mathrm{E}\left\{G_{1}(\boldsymbol{S})\right\}, \ldots, w_{K-1}=\mathrm{E}\left\{G_{K}(\boldsymbol{S})\right\}, \forall p_{\boldsymbol{S}}(\mathbf{s}), \mathbf{s} \in \mathcal{S}\right\}$, respectively. Since the dimension of $W$ reduces to $K$ in this case, the optimal PDF can be obtained as in (27) in this scenario based on similar arguments to those in Appendix B.

\section{REFERENCES}

[1] S. Verdu, Multiuser Detection, 1st ed. Cambridge University Press, 1998.

[2] Y. Shi, R. K. Mallik, and K. B. Letaief, "Randomized power control for two-hop interference channels," IEEE Commun. Lett., vol. 14, no. 12, pp. 1128-1130, Dec. 2010.

[3] N. Tarhuni, M. Elmusrati, and A. Abouda, "Two-state discretized transmit power control for ad-hoc sensor networks," in Proc. 2011 Int. Congress Ultra Modern Telecommun. Control Syst. Workshops, pp. 1-6.

[4] T.-S. Kim and S.-L. Kim, "Random power control in wireless ad hoc networks," IEEE Commun. Lett., vol. 9, no. 12, pp. 1046-1048, Dec. 2005.

[5] M. Elmusrati, N. Tarhuni, and R. Jantti, "Performance analysis of random uniform power allocation for wireless networks in Rayleigh fading channels," Eur. Trans. Telecomm., vol. 20, pp. 457-462, 2009.

[6] _ - "Random power control for uncorrelated Rayleigh fading channels," in Proc. 2007 IEEE Int. Conf. Signal Process. Commun., pp. 360-363.
[7] Y. Zhu, Y. Xu, S. Guan, and J. He, "Connectivity analysis of random power control in wireless sensor networks," in Proc. 2010 IEEE Int. Conf. Commun. Technol., pp. 1248-1251.

[8] D. M. Novakovic and M. L. Dukic, "Evolution of the power control techniques for DS-CDMA toward 3G wireless communication systems," IEEE Commun. Surveys \& Tutorials, vol. 3, no. 4, pp. 2-15, 2010.

[9] S. Koskie and Z. Gajic, "SIR-based power control algorithms wireless CDMA networks: An overview," Dynamics Continuous, Discrete Impulsive Systems B: Appl. Algorithms, no. 10-S, p. 286, 2003.

[10] M. Chiang, P. Hande, T. Lan, and C. W. Tan, "Power control in wireless cellular networks," Foundations and Trends in Networking. NOW Publishers Inc., 2008.

[11] Z. Han, D. Niyato, W. Saad, T. Basar, and A. Hjorungnes, Game Theory in Wireless and Communication Networks: Theory, Models, and Applications. Cambridge University Press, 2012.

[12] K. Akkarajitsakul, E. Hossain, D. Niyato, and D. I. Kim, "Game theoretic approaches for multiple access in wireless networks: a Survey," IEEE Commun. Surveys \& Tutorials, vol. 13, no. 3, pp. 372-395, 2011.

[13] S. Ulukus and R. D. Yates, "Stochastic power control for cellular radio systems," IEEE Trans. Commun., vol. 46, no. 6, pp. 784-798, 1998.

[14] J. Luo, S. Ulukus, and A. Ephremides, "Standard and quasi-standard stochastic power control algorithms," IEEE Trans. Inf. Theory, vol. 51, no. 7, pp. 2612-2624, July 2005.

[15] C. Goken, S. Gezici, and O. Arikan, "Optimal stochastic signaling for power-constrained binary communications systems," IEEE Trans. Wireless Commun., vol. 9, no. 12, pp. 3650-3661, Dec. 2010.

[16] — " "Optimal signaling and detector design for power-constrained binary communications systems over non-Gaussian channels," IEEE Commun. Lett., vol. 14, no. 2, pp. 100-102, Feb. 2010.

[17] — "On the optimality of stochastic signaling under an average power constraint," in Proc. 2010 Allerton Conf. Commun., Control, Comput., pp. 1158-1164.

[18] B. Dulek and S. Gezici, "Detector randomization and stochastic signaling for minimum probability of error receivers," IEEE Trans. Commun., vol. 60, no. 4, pp. 923-928, Apr. 2012.

[19] H. Chen, P. K. Varshney, S. M. Kay, and J. H. Michels, "Theory of the stochastic resonance effect in signal detection-part I: fixed detectors," IEEE Trans. Signal Process., vol. 55, no. 7, pp. 3172-3184, July 2007.

[20] A. Patel and B. Kosko, "Optimal noise benefits in Neyman-Pearson and inequality-constrained signal detection," IEEE Trans. Signal Process., vol. 57, no. 5, pp. 1655-1669, May 2009.

[21] H. Chen, P. K. Varshney, and J. H. Michels, "Noise enhanced parameter estimation," IEEE Trans. Signal Process., vol. 56, no. 10, pp. 50745081, Oct. 2008.

[22] M. Azizoglu, "Convexity properties in binary detection problems," IEEE Trans. Inf. Theory, vol. 42, no. 4, pp. 1316-1321, July 1996.

[23] S. Loyka, V. Kostina, and F. Gagnon, "Error rates of the maximumlikelihood detector for arbitrary constellations: Convex/concave behavior and applications," IEEE Trans. Inf. Theory, vol. 56, no. 4, pp. 1948 1960, Apr. 2010.

[24] R. K. Mallik, R. A. Scholtz, and G. P. Papavassilopoulos, "Analysis of an on-off jamming situation as a dynamic game," IEEE Trans. Commun, vol. 48, no. 8, pp. 1360-1373, Aug. 2000.

[25] M. D. McDonnell, "Is electrical noise useful?" Proc. IEEE, vol. 99, no. 2, pp. 242-246, Feb. 2011.

[26] E. G. Larsson, "Improving the frame-error-rate of spatial multiplexing in block fading by randomly rotating the signal constellation," IEEE Commun. Lett., vol. 8, no. 8, pp. 514-516, Aug. 2004.

[27] — , "Constellation randomization ( $\mathrm{CoRa})$ for outage performance improvement on MIMO channels," in Proc. 2004 IEEE Global Telecommun. Conf., vol. 1, pp. 386-390.

[28] Y. Li, C. N. Georghiades, and G. Huang, "Transmit diversity over quasi-static fading channels using multiple antennas and random signal mapping," IEEE Trans. Commun., vol. 51, no. 11, pp. 1918-1926, Nov. 2003.

[29] C. Lamy and J. Boutros, "On random rotations diversity and minimum MSE decoding of lattices," IEEE Trans. Inf. Theory, vol. 46, pp. 1584 1589, July 2000.

[30] A. Hiroike, F. Adachi, and N. Nakajima, "Combined effects of phase sweeping transmitter diversity and channel coding," IEEE Trans. Veh. Technol., vol. 41, pp. 170-176, May 1992.

[31] X. Ma and G. B. Giannakis, "Space-time-multipath coding using digital phase sweeping," in Proc. 2002 IEEE Global Commun. Conf., vol. 1, pp. 384-388.

[32] H. V. Poor, An Introduction to Signal Detection and Estimation. Springer-Verlag, 1994.

[33] S. A. Grandhi, J. Zander, and R. Yates, "Constrained power control," Wireless Pers. Commun., vol. 1, no. 4, pp. 257-270, 1995. 
[34] C. W. Tan, M. Chiang, and R. Srikant, "Maximizing sum rate and minimizing MSE on multiuser downlink: Optimality, fast algorithms and equivalence via max-min SINR,' IEEE Trans. Signal Process., vol. 59, no. 12, pp. 6127-6143, Dec. 2011.

[35] C. Yang and J. Li, "Mixed-strategy based discrete power control approach for cognitive radios: a matrix game-theoretic framework," in Proc. 2010 Int. Conf. Future Comput. Commun., vol. 3, pp. V3-806V3-810.

[36] T. Heikkinen, "A minimax game of power control in a wireless network under incomplete information," DIMACS Technical Report 99-43, Tech. Rep., 1999.

[37] C. W. Tan, M. Chiang, and R. Srikant, "Fast algorithms and performance bounds for sum rate maximization in wireless networks," IEEE/ACM Trans. Netw., vol. 21, no. 3, pp. 706-719, 2013.

[38] D. W. H. Cai, T. Q. S. Quek, and C. W. Tan, "A unified analysis of max-min weighted SINR for MIMO downlink system," IEEE Trans. Signal Process., vol. 59, no. 8, pp. 3850-3862, 2011.

[39] S. Boyd and L. Vandenberghe, Convex Optimization. Cambridge University Press, 2004.

[40] S. Bayram and S. Gezici, "On the performance of single-threshold detectors for binary communications in the presence of Gaussian mixture noise," IEEE Trans. Commun., vol. 58, no. 11, pp. 3047-3053, Nov. 2010.

[41] J.-J. Xiao and Z.-Q. Luo, "Universal decentralized detection in a bandwidth-constrained sensor network," IEEE Trans. Signal Process., vol. 53, no. 8, pp. 2617-2624, Aug. 2005.

[42] K. E. Parsopoulos and M. N. Vrahatis,"Particle swarm optimization method for constrained optimization problems," in Intelligent Technologies-Theory and Applications: New Trends in Intelligent Technologies, IOS Press, 2002, pp. 214-220.

[43] "CVX: Matlab software for disciplined convex programming, Version 1.21 (April 2011, Build 808).” Available: http://cvxr.com/cvx

[44] R. T. Rockafellar, Convex Analysis. Princeton University Press, 1968.

[45] D. P. Bertsekas, A. Nedic, and A. E. Ozdaglar, Convex Analysis and Optimization. Athena Specific, 2003.

[46] S. Bayram, S. Gezici, and H. V. Poor, "Noise enhanced hypothesistesting in the restricted Bayesian framework," IEEE Trans. Signal Process., vol. 58, no. 8, pp. 3972-3989, Aug. 2010.

[47] C. C. Pugh, Real Mathematical Analysis. Springer-Verlag, 2002.

[48] L. Huang and M. J. Neely, "The optimality of two prices: maximizing revenue in a stochastic network," in Proc. 2007 Allerton Conf. Commun., Control, Comput.

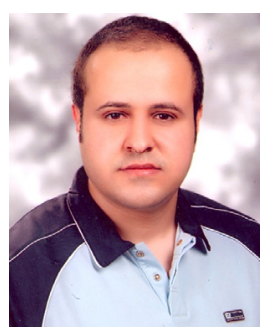

Mehmet Emin Tutay received his B.S. degree in 2008 and M.S. degree in 2010, both from the Department of Electrical and Electronics Engineering, Bilkent University, Turkey. He is currently working towards the Ph.D. degree in the same department. His main research interests are in the fields of statistical signal processing and wireless communications.

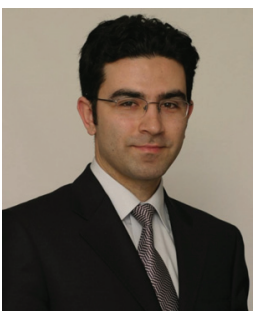

Sinan Gezici (S'03-M'06-SM'11) received the B.S. degree from Bilkent University, Turkey in 2001, and the Ph.D. degree in electrical engineering from Princeton University in 2006. From 2006 to 2007, he worked at Mitsubishi Electric Research Laboratories, Cambridge, MA. Since 2007, he has been with the Department of Electrical and Electronics Engineering at Bilkent University, where he is currently an Associate Professor. Dr. Gezici's research interests are in the areas of detection and estimation theory, wireless communications, and localization systems. Among his publications in these areas, is the book Ultra-wideband Positioning Systems: Theoretical Limits, Ranging Algorithms, and Protocols (Cambridge University Press, 2008). Dr. Gezici is an associate editor for the IEEE TRANS ACTIONS ON COMMUNICATIONS, IEEE WIRELESS COMMUNICATIONS LETTERS, and the Journal of Communications and Networks.

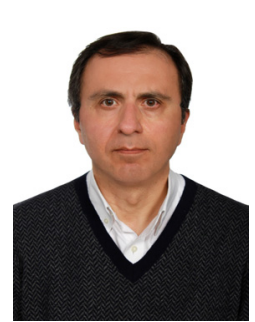

Orhan Arikan (M'91) was born in 1964 in Manisa, Turkey. In 1986, he received his B.Sc. degree in electrical and electronics engineering from the Middle East Technical University, Ankara, Turkey. He received both his M.S. and Ph.D. degrees in electrical and computer engineering from the University of Illinois, Urbana-Champaign, in 1988 and 1990, respectively. Following his graduate studies, he was employed as a Research Scientist at SchlumbergerDoll Research Center, Ridgefield, CT. In 1993, he joined the Electrical and Electronics Engineering Department of Bilkent University, Ankara, Turkey. Since 2011, he has served as the department chairman. His current research interests include statistical signal processing, time-frequency analysis, and remote sensing. Dr. Arikan has served as Chairman of the IEEE Signal Processing Society Turkey Chapter and President of the IEEE Turkey Section. 